\title{
First Order Dominance Analysis: Child Wellbeing in the Democratic Republic of Congo
}

\author{
Malokele Nanivazo
}

Accepted: 8 June 2014/Published online: 10 July 2014

(C) Springer Science+Business Media Dordrecht 2014

\begin{abstract}
This paper performs a multidimensional first order dominance analysis of child wellbeing in the Democratic Republic of Congo (DRC). This methodology allows the ordinal ranking of the 11 provinces of the DRC in terms of their wellbeing based upon the probability of their domination. This empirical application obviates the need to adopt a weighting scheme for the deprivation indicators or to rely on the signs of other crossderivatives for comparison. We execute a bootstrap linear programming algorithm on seven deprivation indicators for three age groups of children derived from the DRC 2007 Standard Demographic and Health Survey. The results reveal widespread disparities in child wellbeing in the DRC.
\end{abstract}

Keywords Welfare - First order dominance - Democratic Republic of Congo · Multidimensional poverty

\section{Introduction}

Questions on who are the poor and how to measure wellbeing have retained the attention of academics and policy makers since the early days of development economics. These questions have sparked a passionate and on-going debate at the nexus of development economics, poverty, and inequality. There is broad consensus, however, that poverty cannot be defined or measured along one dimension; i.e., monetary variables. ${ }^{1}$ Ferreira (2011) states that poverty is characterized by multiple deprivations: low consumption and

\footnotetext{
1 See Kolm (1977), Maasoumi (1986), Atkinson and Bourguignon (1982), Tsui (1999) for other examples of poverty measurement.
}

M. Nanivazo ( $\bowtie)$

United Nations University World Institute for Development Economics Research (UNU-WIDER),

Katajanokanlaituri 6 B, 00160 Helsinki, Finland

e-mail: malokele@wider.unu.edu 
inadequate living standards, in addition to poor health, low life expectancy, limited access to education, knowledge and information, and powerlessness in various domains.

Multidimensional poverty approaches have been used to measure poverty along nonmonetary dimensions of poverty such as social welfare, health, education, and standard of living. These approaches can be divided into two broad categories (1) weighting scheme measurement and (2) robust comparison. Alkire and Foster (2011b) and Alkire and Santos (2011) develop a multidimensional poverty index (MPI) that captures deprivations faced by household members. The MPI, an equally weighted index, is applied to a set of three dimensions (health, education, and standard of living). Ravallion (2010) raises concerns on the need for (1) conceptual clarity on what is being measured, (2) transparency about the trade-offs embedded in the index, (3) robustness of the results, and (4) policy relevance. The robust comparison approach evaluates social welfare functions by comparing welfare achievement among populations without the need of making assumptions on the weight of each deprivation indicator used. It relies on the signs of the second or higher order crossderivatives.

This paper investigates disparities in child wellbeing among the 11 provinces of the Democratic Republic of Congo (DRC). Using three age groups of the children sample, it undertakes a spatial ordinal ranking of the 11 provinces based upon binary welfare indicators. Children are classified based on whether they are deprived in terms of seven deprivation indicators. Ordinal ranking implies that the 11 provinces and the three aggregate areas of the DRC are ranked as better or worse off based upon the probabilities of their domination.

The study utilizes the data from the 2007 DRC Standard Demographic and Health Survey (DHS). The seven deprivations indicators utilized in the empirical analysis are derived from the Bristol deprivation indicators including education, shelter, health, water, sanitation, information, and food (Gordon et al. 2003a, b). The results bring to light the extent of disparities among the 11 provinces in child wellbeing. These results can inform policy makers in crafting policies for appropriate allocation of resources.

The methodology of choice is the multidimensional first order dominance (FOD) approach (Arndt et al. 2012). This approach is based on the theoretical literature on stochastic dominance (see Dyckerhoff and Mosler 1997). Unlike the weighting schemes measurement and robust comparison approach, it does not require a weighting scheme or rely on the signs of the second or other cross-derivatives for comparisons. Theoretically, an FOD comparison between two populations $\mathrm{A}$ and $\mathrm{B}$ can result in three outcomes: A dominates $\mathrm{B}$, B dominates $\mathrm{A}$, or indeterminate domination. Without applying a weighting scheme, the probability of indeterminate domination increases. In the case of clear domination by $\mathrm{A}$ or $\mathrm{B}$, one cannot sufficiently measure the extent of the domination. In addition, one cannot tell which of the welfare indicators creates the domination or whether a small increase (decrease) in the population's share in one of the deprivation indicators can shift the domination from A to B or vice versa.

Empirically, applying a bootstrap approach relaxes these two constraints. The starting point of the bootstrap approach is to select the comparator population from the larger population based on the stratified cluster design of the DHS survey. For each stratum, $K$ samples are randomly drawn with replacement such that $K$ is also equal to the number of population $(N)$ in the primary sampling unit. Such process results in an exhaustive sampling. For this purpose, we utilize a linear programming algorithm to determine FOD across province pairs and run 100 bootstrap iterations, hence unveiling a more detailed perspective on child welfare. 
The organization of this paper is as follows. Section 2 presents a brief literature review on poverty measurement. Section 3 describes the first order dominance approach. Section 4 gives a presentation of the DRC socioeconomic situation, a description of the welfare indicators, and the descriptive statistics. The spatial static and bootstrap comparisons results are reported in Sect. 5. Section 6 concludes that there is strong evidence of disparity in child wellbeing among the 11 provinces of the DRC.

\section{Literature review: poverty measurement}

It is now widely accepted and recognized that poverty is multidimensional. A full picture of poverty cannot be defined in terms of one deprivation such as income deprivation but rather along several deprivations. The seminal work by Sen (1976) focusing on how poverty is measured has triggered a wealth of discussion on poverty measurements and their practicability and implication for welfare.

Popularized by Foster, Greer, and Thorbecke (henceforth FGT 1984, 2010), the unidimensional approach of measuring poverty consists of selecting a cardinal or ordinal welldefined single-dimensional variable and using this variable as the basis for poverty evaluation (Alkire and Foster 2011a). ${ }^{2}$ The FGT class defines poverty as a normalized weighted sum of income shortfalls of the poor or the average poverty in a given population $\left(P_{\alpha}\right)$ (FGT 2010). Where $P_{0}$ is the headcount ratio or the average across the entire population; $P_{1}$ expresses the poverty gap; and $P_{2}$ yields the squared gap measure. There are two main differences between Sen's ordinal measure of poverty and the FGT class based on the squared gap measure $\left(P_{2}\right)$. First, Sen uses a ranking order scheme, which consists of assigning a weight to each poor person until the least poor person is assigned a weight of 1 , whereas, in FGT class measures, $P_{2}$ weights an individual's normalized gap by the normalized gap of the poor (FGT 2010). Moreover, FGT class measures are decomposable by population sub-groups, whereas Sen's measure satisfies the axioms of monotonicity, transfer, and sensitivity. ${ }^{3}$

The multidimensional approach gained prominence in the 1990s with the recognition that poverty is multidimensional, and the advent of UNDP's human development report alongside Sen's capabilities approach. Unlike the unidimensional approach that focuses on a monetary and normative evaluation of poverty, the multidimensional approach mostly focuses on non-monetary dimensions of poverty such as social welfare, health, education, and standard of living. The multidimensional approach is concerned with measuring aggregate poverty rather than identifying the poor (Alkire and Foster 2011a). This strand of literature focuses on measuring poverty by combining various dimensions of deprivation into a single (Alkire and Foster index) or dashboard index (Ravallion index).

Based on Sen's capabilities and functioning approach (1985, 1992), Bourguignon and Chakravarty (2003) aggregate into a single cardinal index the various attributes of an individual's wellbeing and define poverty in terms of a shortfall to that index. Using the case of two attributes or deprivations, Bourguignon and Chakravarty first determine the subsistence or threshold level of each attribute, i.e. the poverty line of each deprivation. The multidimensional poverty index is the average of an individual's aggregate shortfall

\footnotetext{
${ }^{2}$ FGT have used income as their variable of choice. World Bank uses the FGT class for their poverty analysis.

${ }^{3}$ See Alkire and Foster (Alkire and Foster 2011) for thorough description of these axioms
} 
(in the two attributions), raised to the power $\alpha$, over the whole population. ${ }^{4}$ As in the unidimensional case, $\alpha$ is interpreted as a measure of poverty aversion and can take the value of 0 (multidimensional headcount), 1 (multidimensional poverty gap), or higher (interpreted as in the unidimensional case as higher aversion towards poverty). The Bourguignon and Chakravarty multidimensional index depends heavily on the substitutability between the poverty shortfalls in the two attributes with the extent of poverty; i.e., the weight of each attribute.

Duclos et al. (2006) propose a robust dominance multidimensional approach to poverty. They propose a generalization of the unidimensional dominance approach to poverty comparisons developed by Atkinson (1987) and Foster and Shorrocks (1988a, b, c). The authors' approach presents two main advantages. First, it generates poverty rankings that are robust to the choice of poverty index over a broad class of indices eliminating the need for choosing a particular poverty measure. Second, it accounts for the interaction of various dimensions of wellbeing in the measurement of poverty as well as the correlation in the sampled populations. For instance, an increase of income of a poor person would decrease the poverty measure if the poor person is sufficiently deprived in a specific dimension. The authors test their approach on two dimensions (1) per capita household expenditure and (2) nutritional status proxy by child z-scores using the 1993 Vietnam Living Standard Survey. ${ }^{5}$ The results reveal that children living in the rural areas are poorer than those living in the urban areas for any intersection, union, or intermediate poverty frontier.

Alkire and Santos (2011) implement the MPI developed in Alkire and Foster (2011b) to reflect the overlapping deprivations likely to be experienced by household members. The MPI is applied to a set of three dimensions (health, education, and standard of living) measured by 10 indicators. ${ }^{6}$ Each dimension is equally weighted and each indicator is also equally weighted within each dimension (Alkire and Santos 2011). The authors intend to provide information on the joint distribution of the United Nations Millennium Development Goals for contributing in the discussion to accelerate their attainments. They use survey data from 104 countries covering 5.2 billion people ( $78.4 \%$ of the total world population). They find that 1,659 million $(32 \%)$ people in the 104 countries are poor. $^{7}$ Alkire and Santos (2011) estimate that 1,378 million (26\%) live with less than US\$1.25/ day and 2,525 million (48 per cent) with less than US\$2/day. Sub-Saharan Africa has the highest poverty incidence, whereas South Asia the highest number of people living in poverty.

Alkire and Foster (2011a) expand the Bourguignon and Chakravarty multidimensional index in two ways to make the index applicable to: (1) ordinal or categorical variables and (2) more than two attributions or deprivations. Although Alkire and Foster build their MPI on the Bourguignon and Chakravarty index, they propose a dual cut-off method for identifying poor people. The deprivation cut-off $(Z)$ identifies a person being deprived in a dimension $(d)$ when the person's achievement level $(j)$ falls short of the deprivation cut-off $\left(z_{j}\right)$ on that dimension. The poverty cut-off $(K)$ classifies a person as poor if a person's deprivation count $\left(k_{i}\right)$ is equal or above the dimensions considered in the computation of

\footnotetext{
4 The Bourguignon and Chakravarty index satisfies the following axioms: strong focus, weak focus, symmetry, monotonicity, continuity, principle of population, scale invariance, and subgroup decomposability.

5 Their unit of analysis is children younger than 60 months rather than the household.

${ }^{6}$ Health: child mortality and nutrition. Education: years of schooling and child school attendance. Standard of living: electricity, drinking water, sanitation, flooring, cooking fuel, and assets.

${ }^{7}$ Close to 1.7 billion people.
} 
the multidimensional index; i.e., $k_{i} \geq d$. They also use weights or deprivation values, which capture the relative importance of the different deprivations.

Ravallion (2011) questions the need of a single MPI index, the selection of dimensions, the dimension and poverty cut-offs, and the weights assigned to each deprivation. Ravallion proposes the use of prices for forming a composite index in a space defined by all primary dimensions of welfare including commodities called the attainment space and its aggregation called the attainment aggregation. ${ }^{8}$ This is also known as the dashboard index. Contrary to Alkire and Santos (2011) who focus on the joint distribution of deprivations, the author uses the marginal rate of substitution or the relative price in the attainmentaggregation approach. He argues that the marginal rate of substitution captures the tradeoff for reaching one attainment relative to another one. Ravallion suggests developing the best possible distinct measures of the various dimensions of poverty relevant to a given setting - aiming for a credible set of multiple indices rather than a single multidimensional index (2011).

Each of the above poverty measurements has some advantages. Nevertheless, they rely on either a weighting scheme or the signs of the second or higher order cross-derivatives for measuring poverty or identifying the poor. None of these measures can efficiently rank and determine the welfare achievement of a population. The multidimensional first order dominance performs an ordinal ranking of a population and allows comparison of subpopulations both across space and time.

\section{The Multidimensional First Order Dominance}

The multidimensional first order dominance (FOD) approach undertakes an ordinal multidimensional welfare ranking of a given population. FOD is based on multivariate stochastic orderings (see Dyckerhoff and Mosler 1997). Dyckerhoff and Mosler (1997) state that stochastic orderings can occur in two contexts: (1) derived from the structural properties of a stochastic model, and (2) hypothesized between two numeric distributions. Practically, the hypotheses are checked using data and statistical inference. This paper is part of the second context (see also Østerdal 2010). The FOD approach offers two advantages. First, it does not require setting arbitrary weighting schemes across various social welfare achievements. Second, it does not rely on the signs of the second or other cross-derivatives for comparisons.

In the one-dimensional case, assume that we have a finite set of real-value outcomes $X$ such that $\sum f(x)=1$ and $f(x) \geq 0$ for all $x$ in $X$ describing the probability mass function $f$ of the wellbeing distribution of some population. Another population is described by a probability mass function $g$ such that $f$ first order dominates $g$ if any of the following conditions hold:

(a) $g$ can be obtained from $f$ by a sequence of transfers of density from more to less desirable outcomes ${ }^{9}$;

\footnotetext{
${ }^{8}$ These prices can be actual, imputed, or shadow prices for market goods. For non-market goods, the missing prices should be determined a priori or estimated. In the attainment space proposed by Ravallion, weights are prices. Ravallion objects to the use of weights because they are taken to be known and explicit (Ravallion 2011).

${ }^{9}$ In other words, moving some population from $g$ to $f$.
} 
(b) Social welfare is at least as high for $f$ as for $g$ for any nondecreasing additively separable social welfare function such that $\sum_{x \in X} f(x) w(x) \geq \sum_{x \in X} g(x) w(x)$ for any nondecreasing real function $w$;

(c) $F(X) \leq G(x)$ for all $\mathrm{x}$ in $\mathrm{X}$, where $\mathrm{F}(\cdot)$ and $\mathrm{G}(\cdot)$ are cumulative distribution functions corresponding to $f$ and $g .{ }^{10}$

Theoretically, the most intuitive condition is (a) because it guarantees that some population can continually be moved from better to worse outcomes. Then, the dominating population is unambiguously better off.

Turning to the multidimensional case, $f$ and $g$ become multidimensional probability mass function of some population over a finite subset X of $R^{n}$. Then, $f$ FOD $g$ if one of the following conditions holds:

A. $g$ can be obtained from $f$ by a finite number of shifts of density from one outcome to another worse outcome;

B. $\quad \sum_{x \in X} f(x) w(x) \geq \sum_{x \in X} g(x) w(x)$ for every nondecreasing real-valued function $w$;

C. $\sum_{x \in Y} g(x) \geq \sum_{x \in Y} f(x)$ for any comprehensive set $Y \subseteq X$.

Still, condition (A) remains the most intuitive for the same reason as previously stated.

Empirically, the results are derived using a linear programme of a finite set of dimensional cases. Dyckerhoff and Mosler (1997) show that condition (A) can result in a first order dominance using a linear programme for a multivariate discrete case. Arndt et al. (2012) develop a linear programming algorithm, which allows the repeat of the bootstrapping process. This is operationalized using the linear programming technique GAMS, particularly the CONOPT solver develop by Drud (2008). ${ }^{11}$ Here, the process is repeated 100 times. Consequently, we obtain a measure of spatial domination expressed in terms of the percentage of times one province dominates another and interpreted as empirical probabilities.

We undertake two types of comparisons: (1) a static comparison, a one-time comparison of the provinces; and (2) a bootstrap approach, which reveals more information than the one time or static approach. As mentioned, the two comparisons can yield three possible outcomes; population A dominates population B, population B dominates population A, or an indeterminate domination. The bootstrap comparison indicates the probability at which one population solidly dominates another population or vice versa, whereas the static comparison does not give the extent of the domination and may result in an indeterminate domination. Therefore, we can ordinally rank various populations without adopting a weighting scheme of their binary welfare indicators.

\section{Democratic Republic of Congo (DRC): presentation, welfare indicators, and descriptive statistics}

\subsection{Presentation}

The DRC is a Sub-Saharan Africa country with a total area of 2,344,858 square kilometres and a population of 75,507,308 inhabitants (CIA World Fact Book World Bank 2013). ${ }^{12}$

\footnotetext{
${ }^{10}$ Both the uni- and multi-dimensional conditions are derived from Arndt et al. (2012).

11 Arndt et al. (2012) repeat the bootstrapping process 1,000 times.

12 https://www.cia.gov/library/publications/the-world-factbook/geos/cg.html. As of July 2013.
} 
The World Bank (WB) DRC country profile states that "with its immense and extraordinary agricultural and mineral resources, the DRC has the potential to become one of the Africa's richest countries and one of the continent's key engines for growth' (WB 2013). Nevertheless, DRC is classified among the poorest countries in the world.

The precarious condition of the DRC is explained by many factors such as civil wars, political instability, endemic corruption, uncertain legal framework, lack of adequate infrastructure, and substandard macroeconomics policies. Twelve years after the end of the Second Civil War of 1998-2003, the DRC seems to be on path to recovery. Since 2002, the DRC has sustained an average annual growth rate of 6.12 per cent (WB 2013). Many macroeconomic indicators are turning around and there are signs of economic recovery in the agriculture, industry, manufacturing, and services sectors. In 2009, due in part to the 2008 financial crisis, the DRC's GDP growth rate slowed to 2.83 per cent, the lowest level since the end of the civil war. In 2011, the rate of inflation was 2.59 per cent above its level in 2000 .

Despite the overall positive turn of the economy, in 2012, the DRC is ranked as a low income country with a GDP per capita of US\$329.30 and 186 out of 187 in the human development index (HDI). In 2006, 52.76 per cent of its population lived with less than US $\$ 1.25$ a day, and only 45 and 21 per cent had access to water and improved sanitation, respectively (WB 2013). There is a large disparity between the urban and rural areas, towns, and provinces in the DRC.

\subsection{Deprivation Indicators}

This paper empirically analyses child wellbeing in the DRC based on spatial comparisons of the 11 provinces and the three aggregate areas (national, urban, and rural). It focuses on two main questions: (1) what is child poverty and (2) how is child poverty measured? To address the first question, child absolute poverty is defined as a child living in a condition characterized by severe deprivation of basic human needs (Gordon et al. 2003a, 2003b). Gordon et al. (2003a, 2003b) define severe deprivation of basic human needs as circumstances that are highly likely to have serious adverse consequences for the health, wellbeing, and development of children. Here, 'severe deprivation' is measured using the Bristol deprivation indicators: health, education, shelter, information, and water and sanitation (Arndt et al. 2012, Gordon et al. 2003a, 2003b).

1. Sanitation deprivation: Children with no access to any kind of improved latrines or toilets;

2. Water deprivation: Children with only access to surface water for drinking or for whom the nearest source of water is more than a 15 min walking distance from their dwellings;

3. Shelter deprivation: Children living in dwellings with more than five people per room or with no flooring material (e.g., a mud floor);

4. Education deprivation: Children who had never been to primary or secondary school and are not currently attending school; ${ }^{13}$

5. Information deprivation: Children who belong to a household where there is no access to a TV or a radio set;

13 Children were not attending school in 2007, the year when data were collected. 
Table 1 Descriptive statistics of child deprivation indicators (\%)

\begin{tabular}{lllr}
\hline Variables & National & Rural & Urban \\
\hline Children 0 to 5 & & & 43.97 \\
Water deprivation & 74.50 & 94.97 & 54.36 \\
Sanitation deprivation & 63.70 & 69.96 & 62.90 \\
Shelter deprivation & 83.55 & 97.40 & 48.70 \\
Health deprivation & 60.89 & 69.06 & 42.80 \\
Food deprivation & 50.30 & 55.32 & 36.34 \\
Children 6 through 17 & & & 46.30 \\
Water deprivation & 70.19 & 95.38 & 55.53 \\
Sanitation deprivation & 59.19 & 68.77 & 48.74 \\
Shelter deprivation & 79.16 & 96.75 & 13.03 \\
Health deprivation & 60.95 & 70.04 & 29.08 \\
Education deprivation & 22.62 & 29.75 & 63.73 \\
Information deprivation & 48.95 & & 38.53 \\
Children 0 through 17 & & 95.34 & 47.63 \\
Water deprivation & 71.77 & 69.53 & 57.55 \\
Sanitation deprivation & 60.44 & 97.10 & 49.51 \\
Shelter deprivation & 80.69 & 69.54 & \\
Health deprivation & 61.23 & & \\
\hline The columns report & & & \\
\hline
\end{tabular}

The columns report the percentage of children deprived in each indicator. A high percentage means that a large number of children are deprived in the indicator

Source: Author's computation using DHS data

Table 2 Descriptive statistics of individual child deprivation indicators by gender (\%)

\begin{tabular}{|c|c|c|c|c|c|c|}
\hline \multirow[t]{2}{*}{ Variables } & \multicolumn{2}{|l|}{ National } & \multicolumn{2}{|l|}{ Rural } & \multicolumn{2}{|l|}{ Urban } \\
\hline & Female & Male & Female & Male & Female & Male \\
\hline Food deprivation of children under 5 & 47.78 & 52.90 & 51.95 & 58.87 & 41.39 & 44.21 \\
\hline Education deprivation of children 6 through 17 & 25.26 & 20.19 & 33.86 & 26.15 & 14.33 & 11.76 \\
\hline
\end{tabular}

The columns report the percentage of children deprived in each indicator. A high percentage means that a large number of children are deprived in the indicator

Source: Author's computation using DHS data

Health deprivation: Children for whom the nearest health service provider is more than a 15 min walking distance from their dwellings; ${ }^{14}$

6. Food deprivation: Children whose Z-scores for any anthropometrics measures are below -3 standard deviations from the mean of the reference population.

Food deprivation is an index of stunting, wasting, and underweight, which determine child malnutrition (Gordon et al. 2003a, 2003b). The anthropometric measures were

14 The DRC has three types of health providers: public, private, and church hospitals. 
collected on a smaller sample of children aged 0 to 5. Taking this into account, we use food deprivation rather than the individual anthropometric measures.

\subsection{Data and descriptive statistics}

The DRC's standard Demographic and Health Survey (Measure DHS 2007) constitutes the main data source. From the survey, we derive three age groups of the children sample: 1) children aged 0 to 5,2 ) children aged 6 through 17 years old, and 3) children aged 0 through 17. For the age group of children 0 to 5 , we use five out of the seven indicators: shelter, sanitation, health, water, and food. For the second age group, we use six deprivation indicators: sanitation, shelter, water, education, information, and health deprivations. For the larger sample (children 0 through 17), we use the four deprivation indicators, which are common to the two smaller age groups. ${ }^{15}$ Table 1 reports the percentage of children deprived in the seven indicators described above. Table 2 further decomposes the two individual indicators, food and education, by gender.

The first panel of Table 1 reports the percentage of children aged 0 to 5 that are deprived. The national percentage of children 0 to 5 deprived varies between 50.30 per cent (food deprivation) to 83.55 per cent (shelter deprivation). The panel exhibits the disparity between rural and urban areas often observed in most developing countries. A disaggregation of the water deprivation indicator shows a 51 per cent difference between the rural and urban areas. A closer look at the sanitation, shelter, and health deprivation indicators reveal differences of $15.60,34.50$, and $20.36 \%$, respectively. As seen in Table 2, female children are less food deprived than male children in all three areas with this difference most pronounced in rural areas.

Nationally, at least 48.95 per cent of children aged between 6 and 17 are deprived in five out of the six indicators (Table 1, second panel). The education deprivation has the lowest percentage of deprived children, $22.62 \%$. This implies that 22.62 of children aged 6 through 17 have never attended school and did not go to school in 2007. The situation is dire for female children living in the rural areas. Indeed, there is at least $7.71 \%$ difference between the percentages of female children education deprived than male children in the rural areas. Comparatively, shelter deprivation has the highest percentage of children deprived. Indeed, as many as $79.16 \%$ of children live in dwellings with more than five people per room or with no flooring material. The situation is worse in the rural areas where the percentage of children deprived in this dimension reaches $96.75 \%$. Table 1 also reveals that $60.95 \%$ of children are health deprived with a 21.30 per cent difference between the rural and urban.

Note that, the third panel of Table 1 exhibits the same patterns of deprivation as the first and second panels. Nationally, there are more than $61 \%$ of children from 0 through 17 deprived in all four deprivation indicators. More than $71 \%$ of children are both water and shelter deprived.

From the binary indicators, we create three different sets of welfare indicator combinations for each sample. Hence, we have $32\left(2^{5}\right), 64\left(2^{6}\right)$, and $16\left(2^{4}\right)$ combinations for the sample of children 0 to 5, 6 through 17, and 0 through 17, respectively. Table 3 presents the 16 welfare combinations and the shares of children $(0-17)$ by deprivation indicators at the national level and in the 11 provinces of the DRC, respectively. ${ }^{16}$ The first row of

\footnotetext{
15 Children start school at 6 years old.

${ }^{16}$ The capital city of Kinshasa is considered as a province. The welfare combinations for children 0 to 5 and 6 through 17 are available upon request.
} 


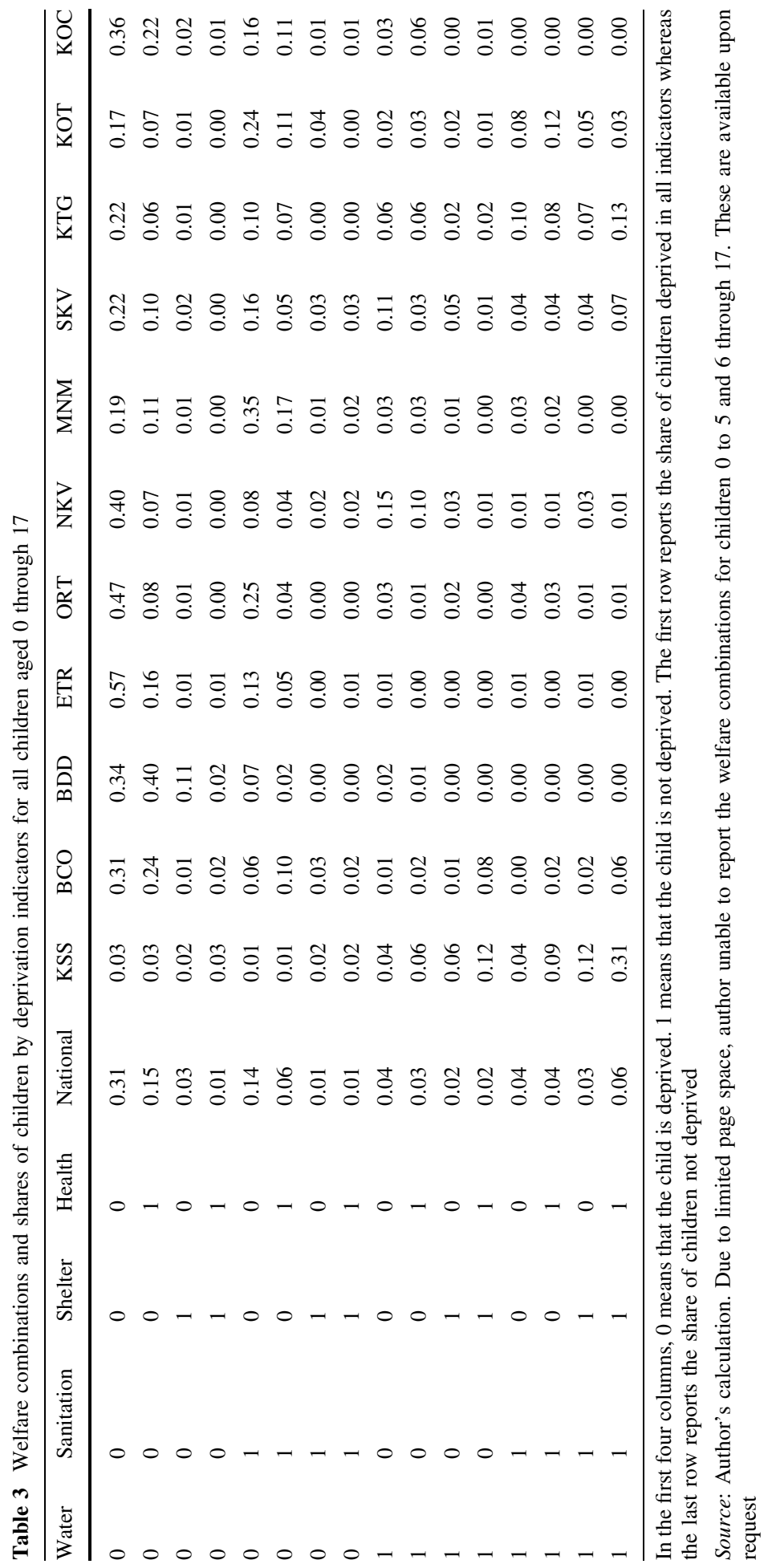


Table 3 shows that Kinshasa (KSS) has the lowest share of children deprived in all four indicators ( 3 per cent) whereas Équateur (ETR) has the highest share of children deprived (57 per cent). Nationally, 31 per cent are deprived in the four indicators. Moving along the column, we move from severe deprivation (deprived in all four indicators) to not deprived in the four indicators. The picture changes drastically. Indeed, Kinshasa has the highest share of children not deprived (31 per cent) compared to four other provinces-Bandundu (BDD), Équateur (ETR), Maniema (MNM), and Kasai-Occidental (KOC)—-that a have the highest percentages of deprived children (zero per cent not deprived) in any indicator.

\section{Results: spatial static and bootstrap FOD}

The spatial FOD comparisons are made across the provinces and the three aggregate areas at a given point of time; i.e., 2007. Tables 4 through 8 report the results of the spatial static and bootstrap FOD comparisons for the three age groups used in the analysis. The row (column) average of Tables 4, 5, 6, 7 and 8 is the probability that a province dominates (or is dominated by) other provinces. Therefore, provinces that are well off would have larger (lower) row (column) averages. This is also captured by the probability of net domination, which is the difference between the row and column averages. Hence, a positive (negative) net domination suggests that the province dominates (or is dominated by) other provinces. From these metrics, provinces and areas are classified in terms of better off, relatively worse off, and worse off provinces.

A ' 1 ' in the static FOD comparison reveals that the child welfare of the row (column) province dominates (is dominated by) the welfare of children in the column (row) province; an empty cell indicates no dominations or the domination is indeterminate. Whereas a ' 1 ' in the bootstrap comparison indicates that the row (column) province dominates (is dominated by) the row (column) provinces 100 per cent of the time, hence revealing a more detailed perspective of children welfare. An empty cell in the bootstrap indicates that the domination of the row (column) provinces is always indeterminate. ${ }^{17}$

\subsection{Children 0 to 5: Static and Bootstrap FOD Results}

Tables 4 and 5 report the results of the spatial static and bootstrap FOD comparisons for children aged 0 to 5. Surprisingly, in both Tables 4 and 5, Kinshasa (KSS) stands among the 11 provinces with higher row averages and net domination; 85 and $81 \%$ respectively. This makes Kinshasa better off than the other provinces. The second best performer is the urban area (URB) with row averages of 62 and $52 \%$ in Tables 4 and 5, respectively.

In the static comparison (Table 4), Katanga (KTG) and the national area (NAT) have row averages of 23 and $15 \%$, respectively. A look of their dominations shows that they have similar domination pattern but Katanga (KTG) dominates North-Kivu whereas the national area's domination over North-Kivu is indeterminate. The remaining provinces have row averages equal to zero except for Kasai-Oriental (8\%). A closer look of its row reveals that it dominates the rural area (RUR). Note that Équateur (ETR) and the rural area (RUR) have the greatest column average compared to other provinces and areas. This implies that Équateur (ETR) and the rural area (RUR) are dominated by more provinces and areas than any other provinces, Moreover, Équateur (ETR) and the rural areas (RUR)

${ }_{17}$ Or it is indeterminate 100 per cent of the time. 


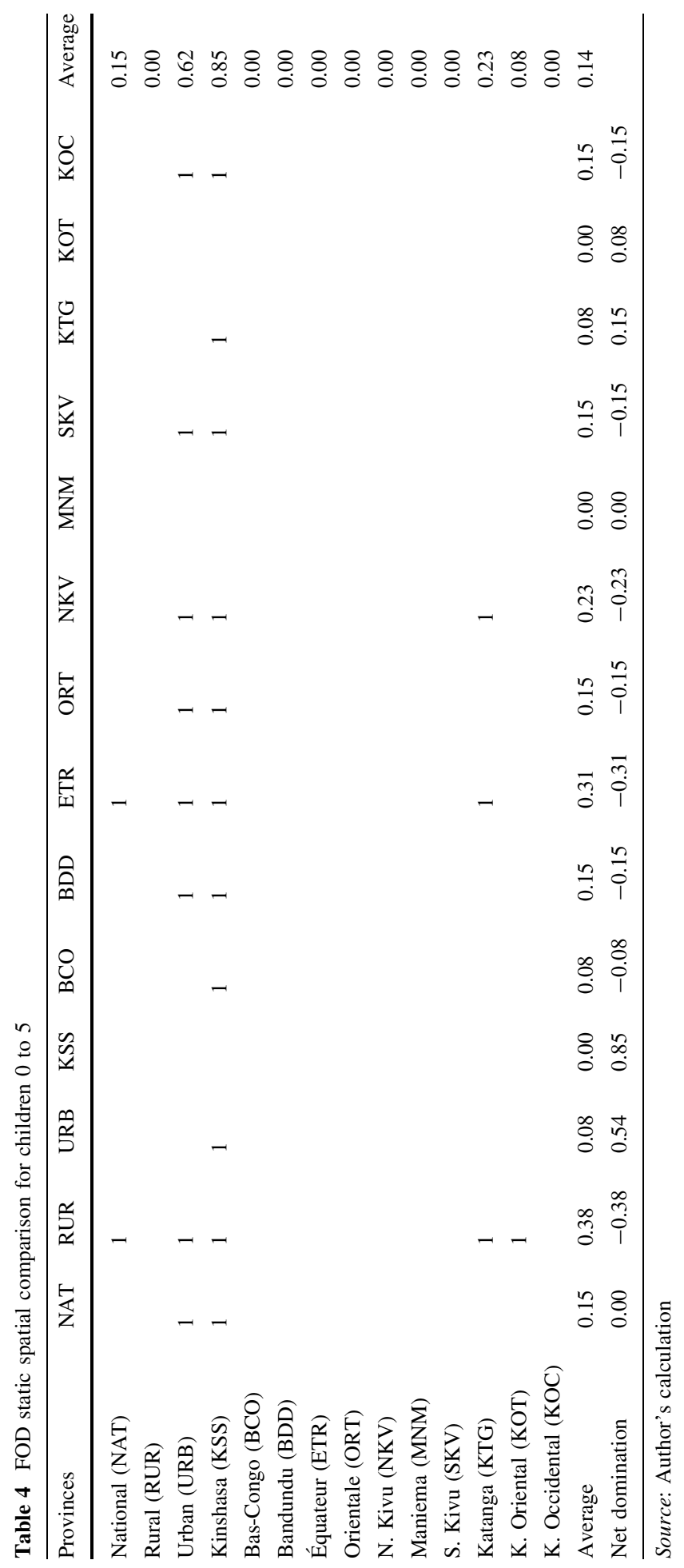




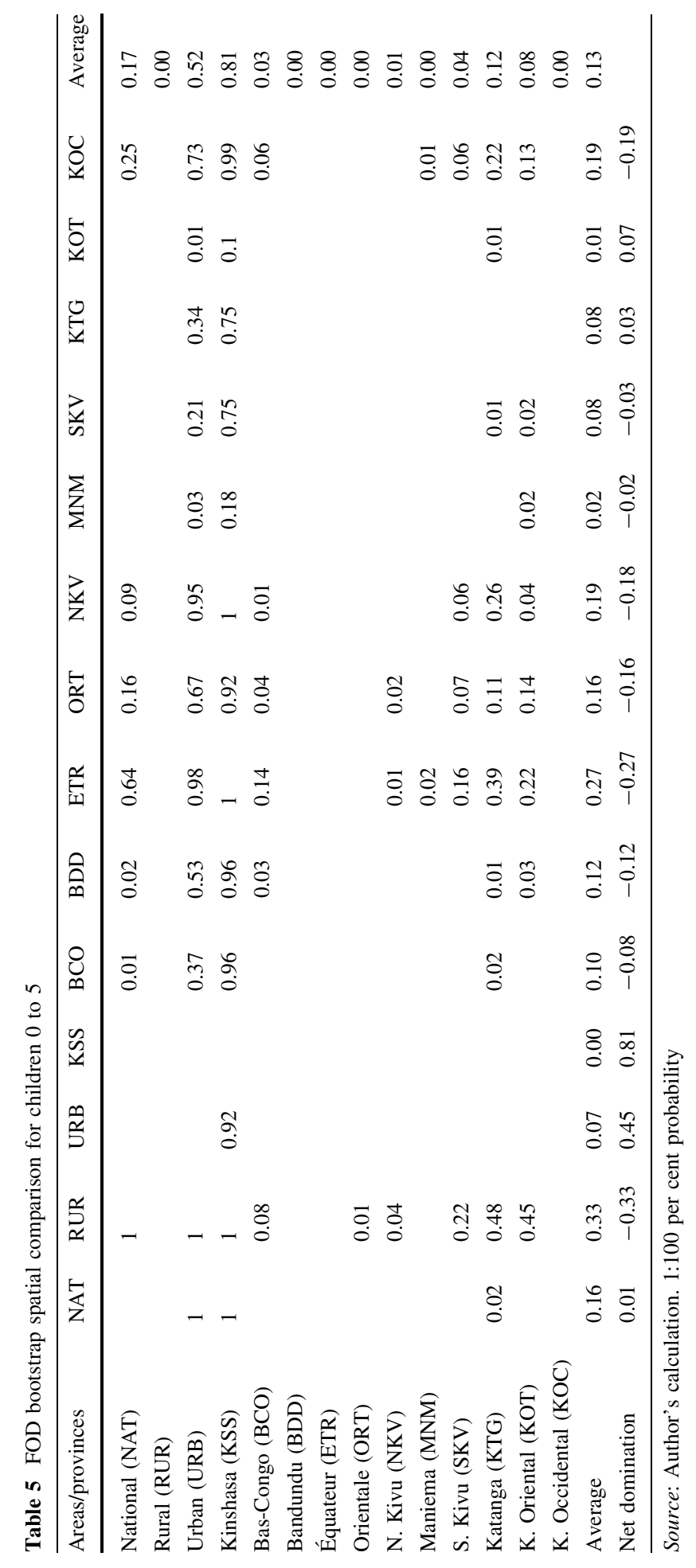


have exactly the same pattern of row domination. As of matter of fact, their dominations over the other provinces and areas are indeterminate.

In the bootstrap FOD (Table 5), among the 11 provinces, Kinshasa is the only province that has row domination of $100 \%$. Indeed, Kinshasa dominates the national and rural areas, Équateur, and North-Kivu $100 \%$ of the time. Unlike the static comparison, the bootstrap FOD has fewer provinces with zero row averages. Nevertheless, there are five provinces with row averages equal to zero in both the static and bootstrap comparisons. These five provinces are Bandundu (BDD), Équateur (ETR), Orientale (ORT), Maniema (MNM), and Kasai-Occidental (KOC). Of these five provinces, Équateur is the worst off with a column average of $27 \%$ and negative net dominations.

Turning to the three aggregated areas, the urban areas are relatively better off than the national and rural areas. This is true for both the static and bootstrap cases (Table 4 and 5). Indeed, the urban areas dominate both the rural and national areas in the static case; its domination reaches $87 \%$ in the bootstrap case.

\subsection{Children 6 through 17: static and bootstrap FOD results}

The static FOD comparison for children between 6 and 17 (Table 6) show that Kinshasa and the urban areas stand out with a row average of 85 and $62 \%$, respectively. Bas-Congo and South-Kivu have row averages equal to 31 per cent whereas the national area (NAT), Katanga (KTG), and Kasai-Oriental (KOT) have row averages equal to $23 \%$. These four provinces and the national area are relatively better off. The remaining provinces and areas have row averages equal to zero except for Maniema (MNM) that has a row average equal to 8 per cent. Nevertheless, these do not tell us which of the provinces is worst off. Comparing Bas-Congo's and South-Kivu's column averages and net dominations, the result reveals that Bas-Congo is better off than South-Kivu. Among the group of provinces with $23 \%$ row averages, Katanga is better off with the lower column average and higher net domination. The remaining provinces have the highest column averages with negative net domination. Here again, Équateur is the lowest performer.

Comparing the aggregate areas (national, rural, and urban), it can be seen that the urban area is better off than the national and rural areas. The urban area dominates the five worse off provinces (Bandundu, Équateur, Orientale, North-Kivu, and Kasai-Occidental) and one relatively better off province (South-Kivu). The urban area is only dominated by Kinshasa. The rural area is the worse off area among the three aggregate areas with a row average of zero per cent. This is due to the fact that the population comprising the rural areas are from lowest performing provinces. ${ }^{18}$

The results from the bootstrap spatial FOD comparison in Table 7 show that, with a row average equal to $86 \%$, Kinshasa is better off than the other provinces. The urban area row average decreases from $62 \%$ in the static case to be $59 \%$ in the bootstrap case. BasCongo's row average decreases from 31 in the static to $21 \%$ in the bootstrap FOD. Nevertheless, Bas-Congo maintains its better off rank compared to South-Kivu and KasaiOccidental. Katanga maintains a row average equal to 23 in the bootstrap FOD. Note that Kinshasa dominates both Bas-Congo and Katanga 96 and $67 \%$ of the time. Comparing Kinshasa to the urban area, we observe that Kinshasa dominates the urban area $93 \%$ of the time. Kinshasa dominates three provinces (Équateur, North Kivu, and Kasai-Occidental) and both the national and rural areas $100 \%$ of the time. Three provinces (Maniema, SouthKivu, and Kasai-Oriental) have row averages ranging from 5 to $16 \%$.

${ }^{18}$ DRC has 21 towns which comprise the urban areas. 


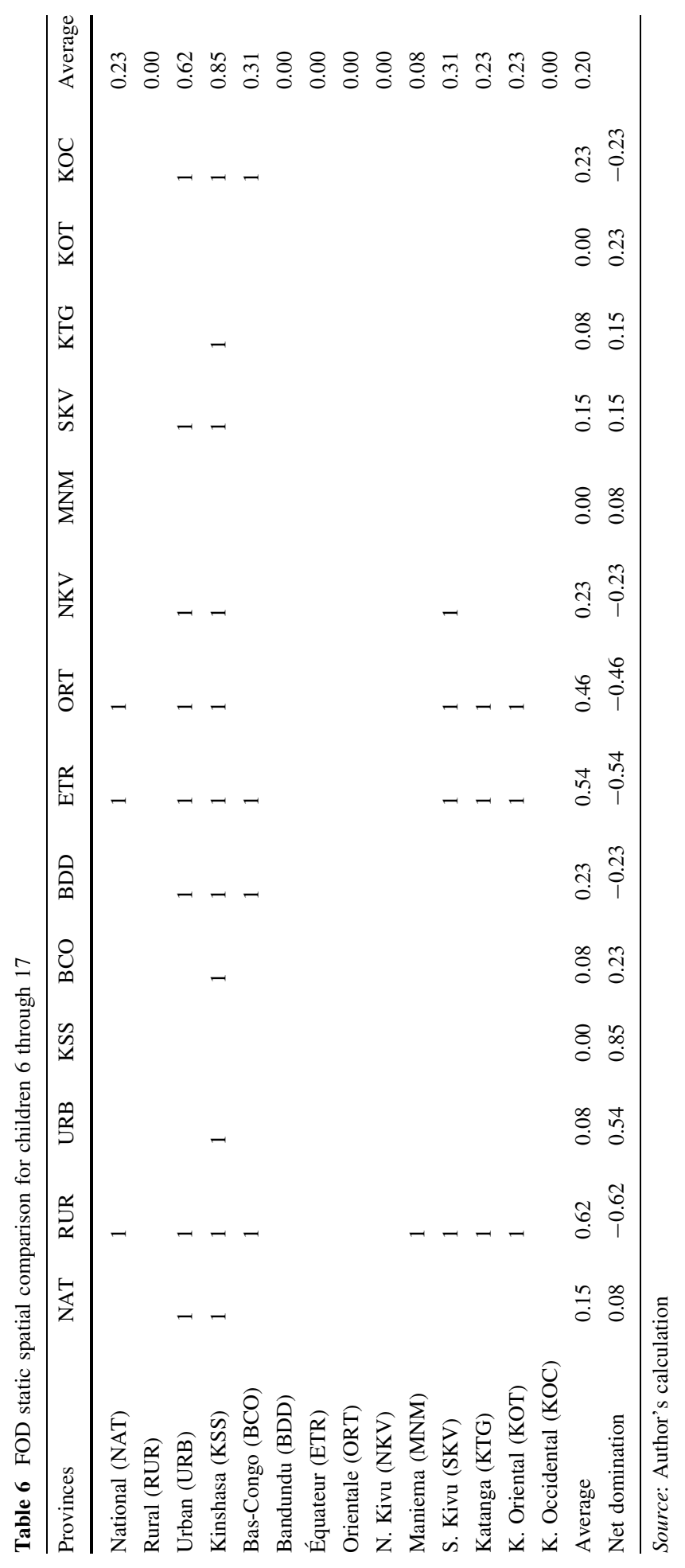




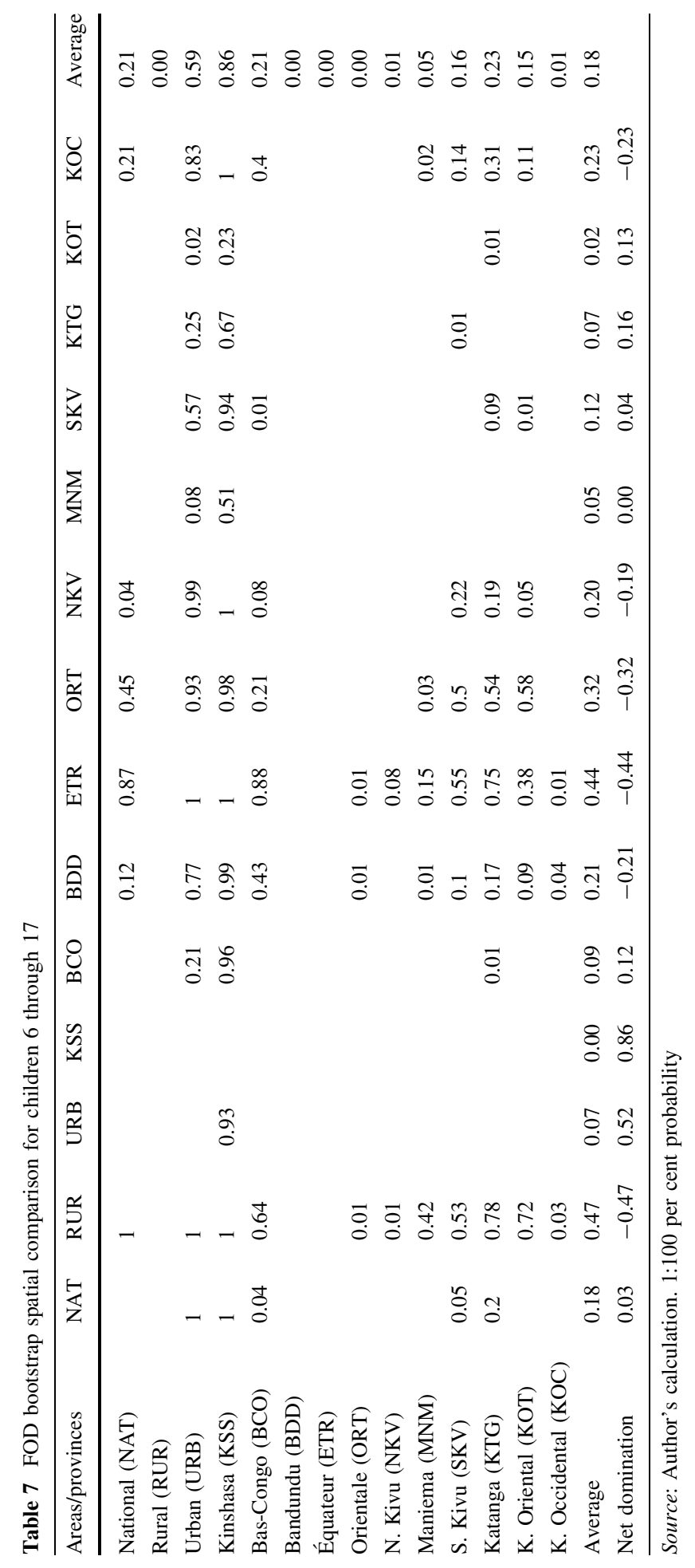


Similarly to the static case, the bootstrap comparison confirms the relatively worse off status of Bandundu, Équateur, Orientale, North-Kivu, and Kasai-Occidental with row averages equal to zero or one per cent. Unlike the static case where their domination over each other is indeterminate, the bootstrap analysis reveals that Orientale dominates Bandundu and Équateur one per cent of the time, whereas North-Kivu dominates Équateur 8 cent of the time. Kasai-Occidental dominates Bandundu and Équateur four and one per cent of the time, respectively. Although three of these provinces have row averages equal to the rural areas; i.e. zero per cent, their column averages makes them better off than the rural area. Indeed, the rural area has the highest column average (47\%) compared to the column averages of Bandundu, Équateur, Orientale, North-Kivu, and Kasai-Occidental. Comparing the column averages of these provinces, Équateur stands out with a column average equal to $44 \%$.

\subsection{Children 0 through 17: static and bootstrap FOD results}

Tables 8 and 9 report the results of the static and bootstrap FOD comparisons for the sample of children 0 through 17 years old. Table 8 reveals that Kinshasa is better off than the ten other provinces with a row average equal to $85 \%$. Surprisingly, Katanga row average is equal to $46 \%$, although this row average is still lower than the urban row average (62\%) but it makes Katanga better off than Bas-Congo and the national areas, which have $23 \%$ row average. The provinces of South-Kivu and Kasai-Oriental both have row averages equal to $31 \%$, whereas North-Kivu and Maniema have row averages equal to $8 \%$. The difference between South-Kivu and Kasai-Oriental is that South-Kivu dominates North-Kivu and Kasai-Oriental dominates Maniema. The provinces of Bandundu, Équateur, Orientale, and Kasai-Occidental and the rural areas have the lowest row averages and have negative net domination making them worst off.

The bootstrap FOD results (Table 9) confirm the better off status of Kinshasa and urban areas with row averages of 87 and $62 \%$, respectively. Katanga maintains its second best position with a row average equal to $38 \%$, whereas Bas-Congo decreases from $23 \%$ in the static analysis to $20 \%$ in the bootstrap analysis. Kinshasa dominates the urban area $91 \%$ of the time, Bas-Congo $96 \%$ of the time, and Katanga $78 \%$ of the time. Katanga dominates Bas-Congo $2 \%$ time whereas its domination over the urban area is indeterminate. South-Kivu and Kasai-Oriental have row average equal to 19 and $24 \%$, respectively. Bandundu, Équateur, and Kasai-Occidental have zero row averages. Although, their dominations are mostly indeterminate, Kasai-Occidental dominates Bandundu $1 \%$ of the time.

Table 10 ranks the provinces and areas based on their probability of net domination in the bootstrap comparison. Based on the metrics of Table 4 through 9, better off (worse off) provinces or areas should have positive (negative) net dominations. Therefore, Kinshasa and the urban area have the highest positive net dominations for all three age groups. This makes them better off. At the bottom of Table 10, we observe that Équateur and the rural area have the lowest negative net dominations. This implies that children living in Équateur and the rural areas are worse off than other children living in the remaining provinces and areas of the DRC. 


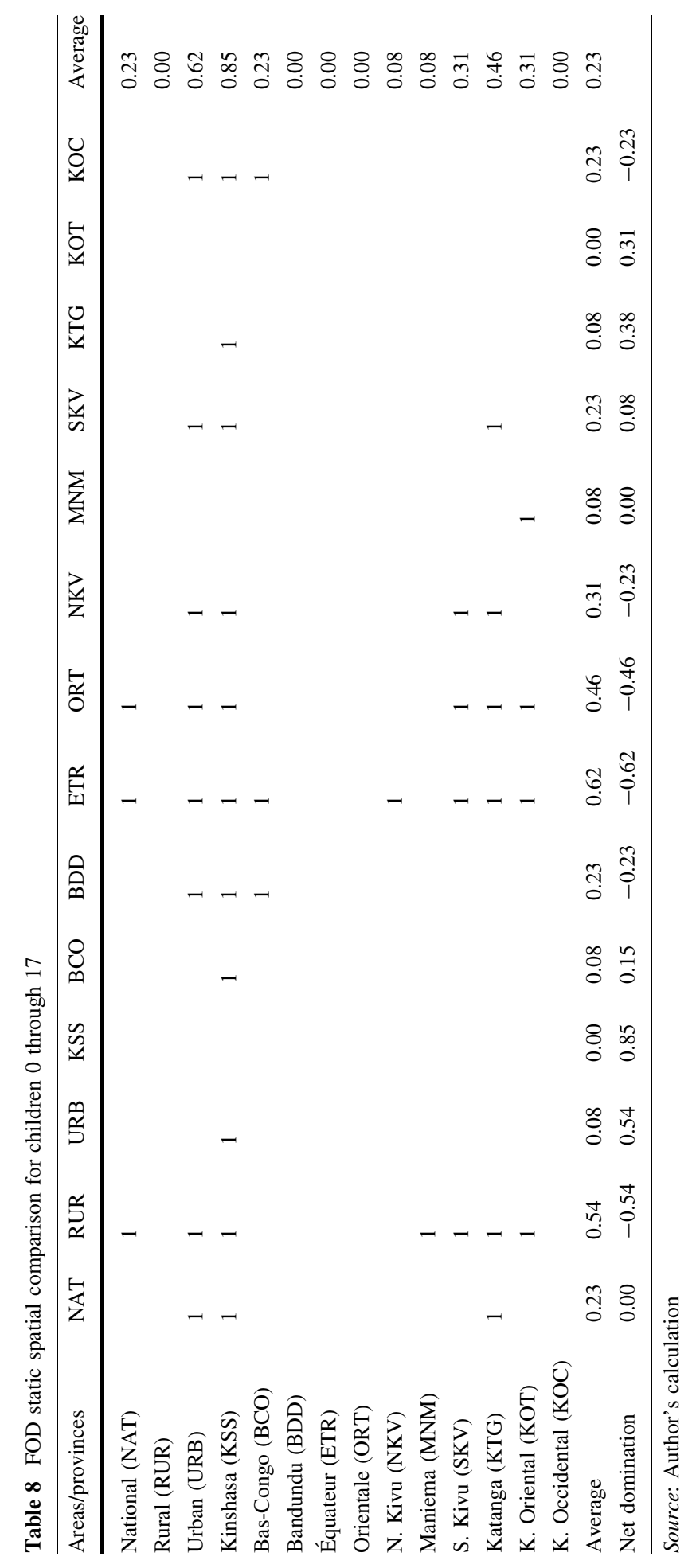




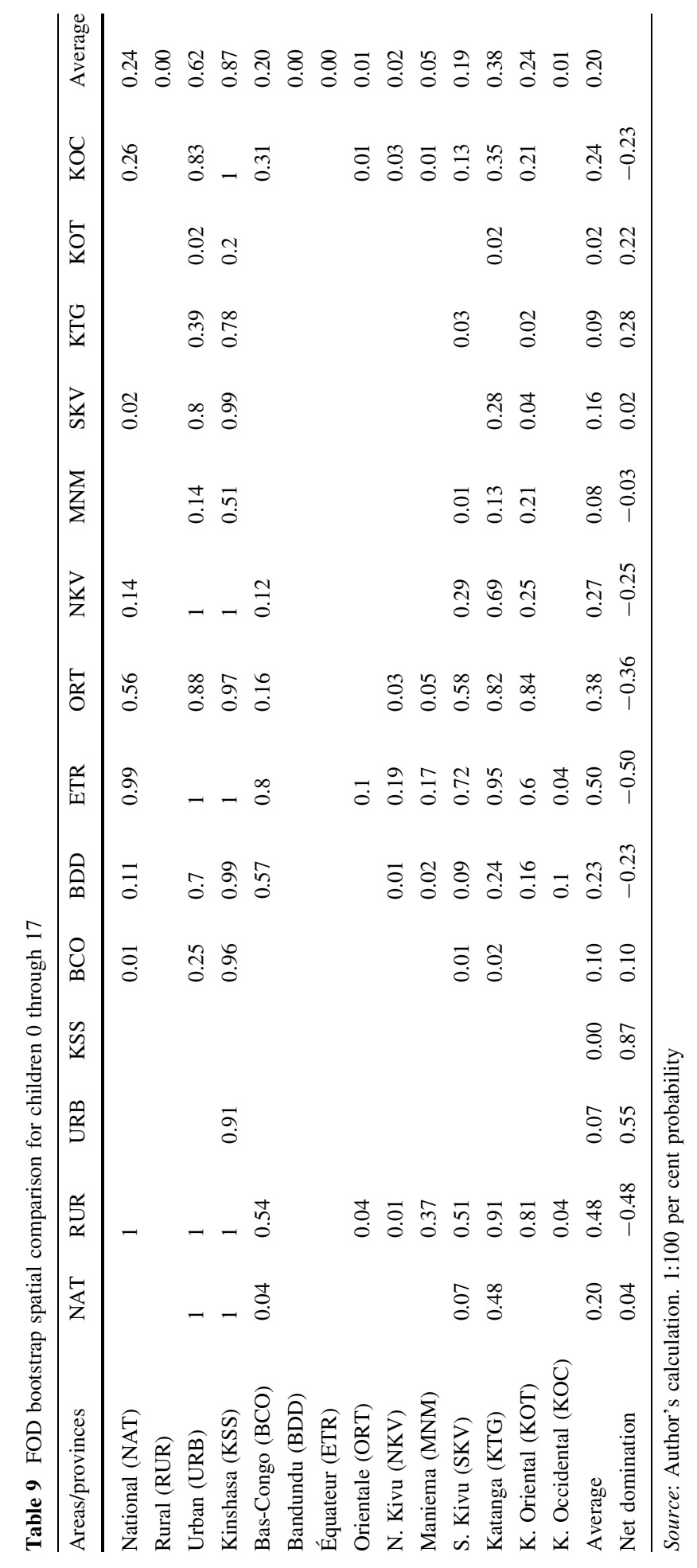


Table 10 Spatial FOD ranking and probability of net domination by area and province

\begin{tabular}{|c|c|c|c|c|c|c|}
\hline \multirow[t]{2}{*}{ Areas/provinces } & \multicolumn{2}{|l|}{ Children 0 to 5} & \multicolumn{2}{|c|}{ Children 6 through 17} & \multicolumn{2}{|c|}{ Children 0 through 17} \\
\hline & Net domination & Rank & Net domination & Rank & Net domination & Rank \\
\hline Kinshasa & 0.81 & 1 & 0.86 & 1 & 0.87 & 1 \\
\hline Urban & 0.45 & 2 & 0.52 & 2 & 0.55 & 2 \\
\hline Kasai-Oriental & 0.07 & 3 & 0.13 & 4 & 0.22 & 4 \\
\hline Katanga & 0.03 & 4 & 0.16 & 3 & 0.28 & 3 \\
\hline National & 0.01 & 5 & 0.03 & 7 & 0.04 & 6 \\
\hline Maniema & -0.02 & 6 & 0.00 & 8 & -0.03 & 8 \\
\hline South-Kivu & -0.03 & 7 & 0.04 & 6 & 0.02 & 7 \\
\hline Bas-Congo & -0.08 & 8 & 0.12 & 5 & 0.10 & 5 \\
\hline Bandundu & -0.12 & 9 & -0.21 & 10 & -0.23 & 10 \\
\hline Orientale & -0.16 & 10 & -0.32 & 12 & -0.36 & 12 \\
\hline North-Kivu & -0.18 & 11 & -0.19 & 9 & -0.25 & 11 \\
\hline Kasai-Occidental & -0.19 & 12 & -0.23 & 11 & -0.23 & 9 \\
\hline Équateur & -0.27 & 13 & -0.44 & 13 & -0.50 & 14 \\
\hline Rural & -0.33 & 14 & -0.47 & 14 & -0.48 & 13 \\
\hline
\end{tabular}

Source: Author's calculation

\section{Conclusion}

The paper's goal is to empirically analyse child wellbeing in the DRC using the multidimensional FOD criterion. The FOD criterion is based on the distributional dominance concept, which is well established in the theoretical literature of stochastic dominance. The FOD criterion eliminates the need for setting arbitrary weighting schemes across various social welfare achievements or relying on the signs of other cross-derivatives for comparisons. Moreover, the FOD criterion delivers robust comparisons of populations particularly for which we have ordinal information at the micro level. This paper sets off the debate on child wellbeing at both the national and provincial levels in the DRC. It is the first empirical analysis that points to the disparity that exists among the 11 provinces of the DRC. It utilizes the 2007 DRC standard Demographic and Health Survey (DHS) to derive seven deprivation indicators based on the Bristol deprivation indicators. For the purpose this paper, we utilize three age groups: children aged 0 to 5, 6 through 17 , and 0 through 17 years old.

The results of the static and bootstrap FOD comparisons provide strong evidence of disparity in child wellbeing among the 11 provinces of the DRC. The spatial static and bootstrap FOD comparisons reveal that children in Kinshasa are better off than children in the other provinces of the DRC. This result holds for the three age groups. Children in the province of Kinshasa are better off than children living elsewhere in the DRC. KasaiOriental is the second best province of the DRC. However, the province of Équateur has been consistently classified as the worst off province of the DRC.

Acknowledgments I would like to thank Channing Arndt for helpful comments on previous drafts of this paper and Ulrik Richardt Beck, Kristi Mahrt, and Isaac Marcelin for their assistance. This paper was 
prepared within the UNU-WIDER 'Growth and Poverty Project' (GAPP), directed by Finn Tarp. It is also published as an UNU-WIDER working paper.

\section{References}

Alkire, S., \& Foster, J. (2011a). Understandings and misunderstandings of multidimensional poverty measurement. The Journal of Economic Inequality, 9(2), 289-314.

Alkire, S., \& Foster, J. (2011b). Counting and multidimensional poverty measurement. Journal of Public Economics, 95(7), 476-487.

Alkire, S., \& Santos, M. E. (2011). 'Acute multidimensional poverty: A new index for developing countries. In Proceedings of the German Development Economics Conference, Berlin 2011, No. 3.

Arndt, C., Distante, R., Hussain, M. A., Østerdal, L. P., Huong, P. L., \& Ibraimo, M. (2012). Ordinal welfare comparisons with multiple discrete indicators: A first order dominance approach and application to child poverty. World Development, 40(11), 2290-2301.

Atkinson, A. B. (1987). On the measurement of poverty. Econometrica, 55(4), 749-764.

Atkinson, A. B., \& Bourguignon, F. (1982). The comparison of multi-dimensioned distributions of economic status. The Review of Economic Studies, 49(2), 183-201.

Bourguignon, F., \& Chakravarty, S. R. (2003). The measurement of multidimensional poverty. The Journal of Economic Inequality, 1(1), 25-49.

Drud, A. (2008). CONOPT. Bagsvaerd, Denmark: ARKI Consulting and Development A/S.

Duclos, J.-Y., Sahn, D. E., \& Younger, S. D. (2006). Robust multidimensional poverty comparisons. The Economic Journal, 116, 943-968.

Dyckerhoff, R., \& Mosler, K. (1997). Orthant orderings of discrete random vectors. Journal of Statistical Planning and Inference, 62(2), 193-205.

Ferreira, F. H. G. (2011). Poverty is multidimensional. But what are we going to do about it? Journal of Economic Inequality, 9(3), 493-495.

Foster, J., Greer, J., \& Thorbecke, E. (1984). A class of decomposable poverty measures. Econometrica, 52(3), 761-766.

Foster, J., Greer, J., \& Thorbecke, E. (2010). The Foster-Greer-Thorbecke (FGT) poverty measures: 25 years later. The Journal of Economic Inequality, 8(4), 491-524.

Foster, J. E., \& Shorrocks, A. F. (1988a). Poverty orderings. Econometrica, 56(1), 173-177.

Foster, J. E., \& Shorrocks, A. F. (1988b). Poverty orderings and welfare dominance. Social Choice and Welfare, 5(2-3), 179-198.

Foster, J. E., \& Shorrocks, A. F. (1988c). Inequality and poverty orderings. European Economic Review, 32(2), 654-661.

Gordon, D., Nandy, S., Pantazis, C., Pemberton, S., \& Townsend, P. (2003a). Child poverty in the developing world. Bristol: The Policy Press.

Gordon, D., Nandy, S., Pantazis, C., Pemberton, S., \& Townsend, P. (2003b). Using Multiple Indicator Cluster Survey (MICS) and Demographic and Health Survey (DHS) Data to Measure Child Poverty. In UNICEF MICS conference.

Kolm, S. C. (1977). Multidimensional egalitarianisms. The Quarterly Journal of Economics, 91(1), 1-13.

Maasoumi, E. (1986). The measurement and decomposition of multi-dimensional inequality. Econometrica, 54(4), 991-997.

Measure DHS (2007). Democratic Republic of Congo Demographic and Health Surveys. In ICF International, Calverton, 2013. Accessed 27 Sept 2013.

Østerdal, L. P. (2010). The mass transfer approach to multivariate discrete first order stochastic dominance: Direct proof and implications. Journal of Mathematical Economics, 46(6), 1222-1228.

Ravallion, M. (2010). Mashup indices of development. Policy Research Working Paper 5432, Washington: World Bank.

Ravallion, M. (2011). On multidimensional indices of poverty. The Journal of Economic Inequality, 9(2), $235-248$.

Sen, A. (1976). Poverty: An ordinal approach to measurement. Econometrica, 44, 219-231.

Sen, A. K. (1985). Commodities and capabilities. Professor Dr. P. Hennipman Lectures in Economics', New York: North-Holland.

Sen, A. (1992). Inequality reexamined. Oxford: Clarendon Press.

Tsui, K. Y. (1999). Multidimensional inequality and multidimensional generalized entropy measures: An axiomatic derivation. Social Choice and Welfare, 16(1), 145-157.

World Bank (2013). Democratic republic of congo overview. http://www.worldbank.org/en/country/drc/ overview. Accessed 22 Jan 2014. 